\title{
PDGFRA Mutation Analysis
}

National Cancer Institute

\section{Source}

National Cancer Institute. PDGFRA Mutation Analysis. NCI Thesaurus. Code C158944.

A procedure used to detect and identify mutations in the PDGFRA gene. 